English. Besides the acidulous wines of the former, the use of vegetables and vegetable soups is large in France. The contrast between the inhabitants of the two countries on this point is considerable.

Digestive derangements which affect the constitution of the urina potus indicate more deep-seated mischief, either in the stomachic or the renal functions, than derangements of the urina sanguinis.

Urine which immediately on being roided gives out a sensible smell of ammonia, generally indicates that the vital powers have suffered declension; that disease has become chronic; that the patient is past middle life, or is prematurely aged, \&c.

In arthritic and rheumatic cases, the mineral and vegetable acids are carefully to be shunned. Among the latter the oxalic is the most objectionable; next, the malic; then, the tartaric, citric, and acetic. There can be little doubt that these acids act injuriously by their astringent effect on the cutaneous and mucous surfaces, by their thus interfering with their own elimination and that of the uric and lactic acids thereby loading the blood with acidulous principles; whence follows that peculiar irritative condition of the nerves, constituting local affections, such as sciatica, lumbago, gout, or the systemic disturbance of rheumatic fever. It is amazing how difficult it is to rid the blood of this acidulous diathesis (if the expression may be used) when once it has been formed. The excernents seem to find it a peculiarly hard task to eliminate acids. Years of rigid attention to the dietetic ingesta are necessary. Hence the rarity of radical cures of gout and rheumatism.

Ravenscourt Villas, Hammersmith, 1851.

\section{ON A POINT OF UTERINE PATHOLOGY.}

\section{Br WALTER C. DENDY, EsQ., M.R.C.S.}

THE physiology of the placenta is a subject of the highest interest, both with reference to fotal development and to maternal welfare. To its abnormal or unhealthy condition may be referred many of those deranged actions which often prove so detrimental and perilous to the mother, especially during the progress of parturition.

The morbid states of its vascular portion will constantly interfere with the economy of the footus, especially with its respiratory apparatus and its nutrition; its prosperous development will thus be at once checked, and itself, and subsequently its mother, be placed in difficulty or danger.

It is, however, to the maternal or cellular portion of the placenta that I now briefly refer, and this especially regarding the parturient uterus.

The microscope will gradually unfold the morbid anatomy of the placenta, the various derangements which its cellular tissue is undergoing, and will probably illustrate the influence which chronic inflammation, disintegration of coagula, formation of cyst, \&c., possess over the normal connexion of the ovum with the uterus. I do not here, however, at present enter into the special pathology of the uterine and placental cells, or of the decidua, so intimately concerned in placental adhesion. There are, howcver, two conditions of the parturient uterus which, I believe, are very intimately associated with placental pathology: I allude to protracted or inefficient uterine efforts, to which the term lingering labour has been applied; and the inverted or irregular action of the uterine fibres subsequent to delivery.

If the placental cells be obstructed by fatty or other degeneration, a low organic power is induced, the foetus is of course imperfectly nourished, and it will probably dwindle, or be prematurely dislodged from the uterine attachments.

For this result, however, the tissues must be extensively disorganized; for there will ensue a degree of vicarious action in the healthy placental lobules, in aid of those which are dis. eased.

To induce, however, those unhealthy states to which I have alluded, a morbid change in one or two lobules may be sufficient, especially if this contamination extend to the tissue or lining membrane of the uterus.

We know that whenever a tissue is inflamed or diseased, there will often be a sort of instinctive reluctance to motiona dread of its consequence; as if the organ were a ware of the pain or unensiness which motion would induce: hence the organic apathy and the suspension of action. The degeneration or disease of the cellular portion of the placenta, must constantly be productive of some morbid change in those tissue intimately associated with it, and vice versat: one of these changes being subacute inflammatory action and adventitious adhesion.

Now we are all aware that systemic or constitutional debility in the mother, may often be the source of inefficient muscular action, as well as of insufficient fœtal nutrition, by which labour may either be protracted or prematurely induced; but, from the frequent association of irregular uterine action with placental derangement, I believe they may more often be considered as cause and effect than we have yet been able to rove.

The last three cases of morbidly adherent placenta which have occurred to me have been in ladies who have frequently aborted, and in whom labours have almost invariably been lingering, and in two of which hour-glass contraction has been combined with morlid adhesion. The last case of this irregular contraction occurred to me three weeks since, and has revived the reflections that have led to these brief propositions.

Mrs. L-, a lady of fair complexion, yet of very firm and healthy constitution, who has miscarried three times, and in whom every complete labour has been marked by some unusual circumstances, had been indisposed from Friday night until the following Tuesday; the membranes had suddenly yielded withont effort. In this interval there was no actual labour pain; the expression of the patient being that she felt very odd and queer, and experienced very unusual sensations in the abdomen. On Tnesday at noon, uterine effort supervened, and late in the evening the child was born. The extraction of the feet was, however, even difficult; for although, for several reasons, $I$ allowed the uterns to expel its contents without much tractile effort of my own, I was obliged to relieve the feet from a very firm contraction, which girt the ankles with extreme force. The funis was very fragile, and could have been easily torn by traction; and as $I$ at once discovered a close hour-glass contraction, I directly proceeded to overcome it. On passing the hand into the superior cavity, the wrist was girt by an excess of power, which those who have been subjected to this grasp will not soon forget. The placenta was firmly adherent, and required very careful detachment, my fingers being constantly enmeshed, as it were, by membranous bands. The lady recovered without any morbid symptom, two very small placental lobuli passing on the fourth day, somewhat decomposed.

The character of pains in lingering labour is ever indicative of abnormal action, deficient or irregular; and it is probable I think, that the fibres about the fundus uteri, where morbid adhesion equally occurs, are perfectly apathetic, when the unnatural labour depends on the morbid condition of the cells to which I have alluded, for it occurs very of ten in women whose robust frames and constitutions would promise vigorous and efficient uterine action.

In those cases which are of a nervous or asthenic character, occurring in women of weak or lax fibre, a full dose of opium given to induce repose will often excite efficient labour pains, by controlling irregular action; but where a morbid state of the tissues themselves exists, opium possesses not this influence.

When the foetns is expelled from a uterus thus morbidly associated, the same irregularity will be displayed. The innervation which would otherwise be diffused over the whole fibrous tissue of the uterus, is concentrated in those portions which still retain their power and energy; these act with undue force, and hence we may explain the almost Titanic resistance which the ring offers to our efforts to introduce the and.

Hour-glass contraction has often been referred to injudicious traction of the child in the absence of nterine pains. This officiousness is far more frequently the cause rather of inertness, of inefficient uterine contraction than the grasping ring of hour-glass contraction; and we know that some of the most obstinate cases have occurred, not only where the birth has been left solely to natural effort, but even before the expulsion of the child from the womb.

I have written these brief remarks chiefly with a view of drawing attention to the pathology of the uterine decidua.

The Glanders in the Human Subject. $-\mathbf{A}$ French veterinary surgeon died lately of glanders at Vats, (department of Charente.) Unfortunately some of the farcy matter from horses which the surgeon had attended, and caused to be killed, found its way into his own eye; and in spite of the most energetic treatment the poor man died in a few days. It is to be hoped that this case will serve as a warning to those who may have the care of horses affected with glanders; they cannot be too cautious. 